\title{
APLICAÇÃO DA FLOCULAÇÃO MICELAR ADSORTIVA PARA REMOÇÃO DO CORANTE DIRECT YELLOW 27
}

\author{
R. P. F. MELO ${ }^{1}$, E. L. BARROS NETO ${ }^{1}$, T. N. CASTRO DANTAS ${ }^{1}$, A. G. CÂMARA ${ }^{1}$ \\ ${ }^{1}$ Universidade Federal do Rio Grande do Norte, Departamento de Engenharia Química \\ E-mail para contato: ricardo_pfm@yahoo.com.br
}

\begin{abstract}
RESUMO - O setor têxtil é responsável por gerar uma elevada carga de efluente devido ao elevado consumo de água nos processos de tingimento. Uma alternativa para tratá-lo é a Floculação Micelar Adsortiva (FMA), que envolve a atração de um cátion metálico para superfície de micelas de tensoativo aniônico, formando flocos hidrofóbicos capazes de atrair compostos orgânicos dissolvidos no meio. Neste trabalho a FMA foi realizada utilizando como tensoativo o sabão base e o cálcio como metal para remover o corante Direct Yellow 27, analisando três parâmetros: a concentração de tensoativo, a temperatura e o $\mathrm{pH}$. Os resultados mostram que o processo pode ser utilizado para o tratamento do efluente contendo corante, observando que a remoção alcançou 97,58\%. O coeficiente de distribuição, razão da massa de corante adsorvido pela massa em solução, alcançou aproximadamente 10 , mostrando a afinidade do corante pelo floco.
\end{abstract}

\section{INTRODUÇÃO}

A indústria têxtil está entre as maiores poluidoras do mundo devido, principalmente, à presença de corantes, compostos orgânicos de estrutura química complexa, os quais pertencem a um conjunto de substâncias agressivas que degradam de modo intenso o meio ambiente. Além disso, o efluente gerado é formado por compostos complexos e de grande variedade como, por exemplo, agentes sequestrantes, carreadores de corantes, agentes dispersantes, etc. (Arslan-Alaton et al, 2008). Portanto, considerando o volume descarregado e a composição do efluente, o efluente têxtil é considerado como o mais poluente dentre todos os setores industriais (Tehrani-Bagha et al, 2010).

No caso de tingimento com corante direto, este é fixado à fibra de algodão por meio de um processo que utiliza banhos contendo sais. Conseqüentemente, nos processos de lavagem que ocorrem após o tingimento estes sais são solubilizados, promovendo a retirada do principal fator que permite a 


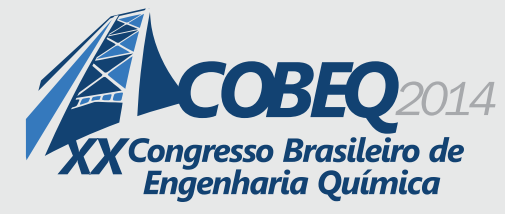

fixação do corante a fibra. Dessa forma, o tingimento com corantes diretos possuem uma fixação baixa aos tratamentos úmidos.

Diante da problemática da indústria têxtil com seu efluente muitos estudos têm sido direcionados para o desenvolvimento de novas alternativas de tratamento. Dentre os processos aplicados até então, pode-se citar os processos oxidativos avançados (Muruganandham e Swaminathan, 2006), coagulação-floculação (Lau et al, 2014), adsorção (Zhang et al, 2014) e extração por ponto nuvem (Purkait et al, 2006), todos apresentam resultados favoráveis na remoção do poluente das águas residuais.

Entre os muitos processos estudados para o tratamento de efluentes, existe a Floculação Micelar Adsortiva, esta técnica baseia-se na reação de tensoativos iônicos com cálcio para formar um sal insolúvel que permanece no meio na forma de pequenos flocos, atraindo compostos orgânicos solúveis devido ao caráter hidrofóbico do floco proporcionado pelo tensoativo. Esses flocos são subseqüentemente separados da solução por uma etapa de filtração, que retira o floco contendo a maior parte do corante que se encontrava em solução. Fatores como concentração de tensoativo e cálcio, temperatura e $\mathrm{pH}$ mostram-se importantes para eficiência do processo.

Diante da necessidade do desenvolvimento de novos processos para o tratamento do efluente contendo corante, este trabalho propõe aplicar uma metodologia inovadora ao utilizar tensoativos aniônicos através da floculação micelar adsortiva para retirar o corante de efluentes têxteis, visando, principalmente, determinar as melhores condições de processo.

\section{MATERIAIS E MÉTODOS}

\subsection{Materiais}

Sabão base foi utilizado como tensoativo aniônico, sendo formado a partir de $5 \%$ de óleo de coco e $95 \%$ de sebo bovino, peso molecular médio $289 \mathrm{~g} / \mathrm{mol}$, balanço hidrofílico-lipofílico 20,4 e o grupo cabeça é COONa. O corante Direct Yellow 27 de peso molecular 662,62 $\mathrm{g} / \mathrm{mol}$ e fórmula molecular $\mathrm{C}_{25} \mathrm{H}_{20} \mathrm{~N}_{4} \mathrm{Na}_{2} \mathrm{O}_{9} \mathrm{~S}_{3}$ foi utilizado neste trabalho. A estrutura química do Direct Yellow 27 é apresentada na Figura 1.

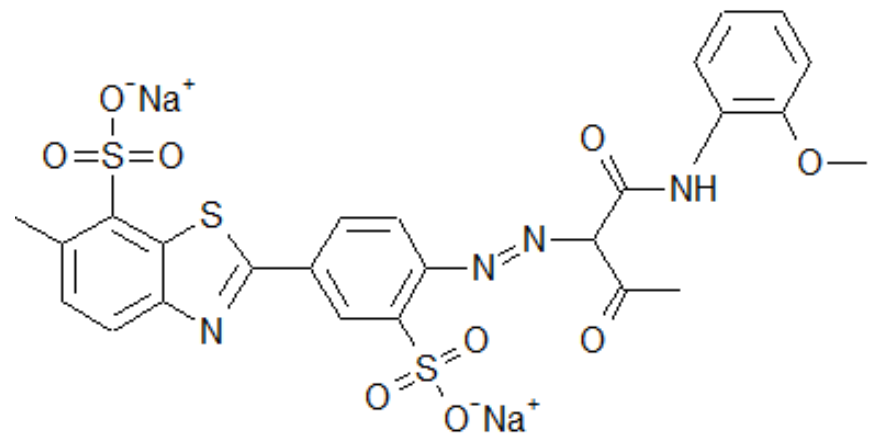

Figura 1 - Estrutura química Direct Yellow 27. 


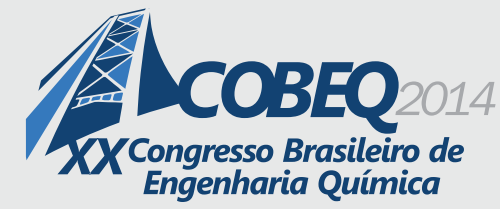

O cálcio aplicado na FMA foi obtido a partir de uma solução de $\mathrm{CaCl}_{2}$, sendo a concentração de $\mathrm{Ca}^{2+}$ em solução 3600 ppm.

\subsection{Procedimento Experimental}

Os experimentos foram realizados no Water Separability Tester (Marca: Koehler Instrument Company Inc., Modelo: K39396), provido de banho termostático e agitador para homogeneização. As amostras foram preparadas com concentração fixa de corante de $100 \mathrm{ppm}$. Nos experimentos em que foi estudado o efeito da concentração de tensoativo foram usadas concentrações de 130, 170, 220, $260,290,330,360,375,390,520$ e 650 ppm, as concentrações de cálcio nas amostras sempre foram metade da concentração de tensoativo. Todas as amostras são previamente colocadas no banho termostático, sendo avaliadas as temperaturas de $30,35,40,45,50$ e $60{ }^{\circ} \mathrm{C}$.

O tensoativo utilizado foi previamente triturado e adicionado à solução contendo corante, sendo dissolvido na solução, quando submetido a uma agitação de $1.000 \mathrm{rpm}$ até garantir a completa solubilização. Depois, a solução de cálcio foi adicionada para promover a formação dos flocos, totalizando um volume final da amostra de $100 \mathrm{~mL}$. Imediatamente após a adição do cálcio, inicia-se a agitação das amostras numa rotação de $300 \mathrm{rpm}$ durante três minutos e, em seguida, esta agitação é reduzida para $150 \mathrm{rpm}$ por um período de 2 minutos. Cessada a agitação, as amostras permanecem em repouso durante trinta minutos para garantir o equilíbrio.

Passado o tempo de repouso, as amostras são filtradas utilizando filtro analítico AP40 em microfibra de vidro, sem resina, poro $0,7 \mu \mathrm{m}$. O filtrado é analisado em espectrofotômetro de absorção molecular (Marca: Varian, Modelo: Cary 50 Conc) e em espectrofotômetro de absorção atômica (Marca: Varian, Modelo: AA240) para determinar a concentração de corante e cálcio finais, respectivamente.

Os experimentos em que foi estudado o efeito do $\mathrm{pH}$ o procedimento foi o mesmo, diferenciando-se apenas a etapa de ajuste desta variável, a qual foi realizada antes da adição de cálcio às amostras, sendo utilizadas soluções de ácido clorídrico e hidróxido de sódio neste procedimento. Os valores de $\mathrm{pH}$ aplicados foram $7,8,9,10,11,12 \mathrm{e} 13$, todos na temperatura de $30^{\circ} \mathrm{C}$.

\subsection{Determinação da eficiência de remoção e coeficiente de distribuição}

As equações utilizadas para determinação da eficiência de remoção de corante e do coeficiente de distribuição são dadas pelas Equações 1 e 2, respectivamente.

$$
\begin{aligned}
& \% \text { remoção }=\frac{C_{D Y 27, \text { inicial }}-C_{D Y 27, \text { final }}}{C_{D Y 27, \text { inicial }}} \times 100 \\
& K=\frac{m_{D Y 27 \text { no floco }}}{m_{D Y 27 \text { na fasediluída }}}
\end{aligned}
$$




\section{RESULTADOS E DISCUSSÕES}

Esta seção é dividida em três partes. Na primeira parte, será discutido o efeito da concentração de tensoativo e temperatura sobre a eficiência de remoção de corante. A avaliação do coeficiente de distribuição do processo dar-se-á na segunda parte. Por último, o efeito do pH na extração do corante será analisado.

Os valores de concentração de tensoativo foram escolhidos na faixa de 130 a 650 ppm, visto que em concentrações inferiores a 130 ppm não houve extração do corante e, acima de 650 ppm a remoção permaneceu praticamente constante. Além disso, estabeleceu-se que as amostras teriam concentração de corantes fixas, pois em ensaios iniciais observou-se um efeito não significativo desta variável no processo.

\subsection{Eficiência de remoção de corante}

De acordo com os resultados obtidos verificou-se que a FMA é um processo apropriado para remoção do corante do efluente sintético. A eficiência de remoção foi avaliada para diferentes temperaturas e concentrações de tensoativo, com o propósito de determinar a melhor condição de operação. Analisando os dados, nota-se que a concentração de tensoativo tem efeito significativo na FMA. Os resultados alcançados para diferentes temperaturas e concentrações de tensoativo estão na Figura 2. Todos os pontos da Figura 2 são resultados da média de ao menos duas repetições.

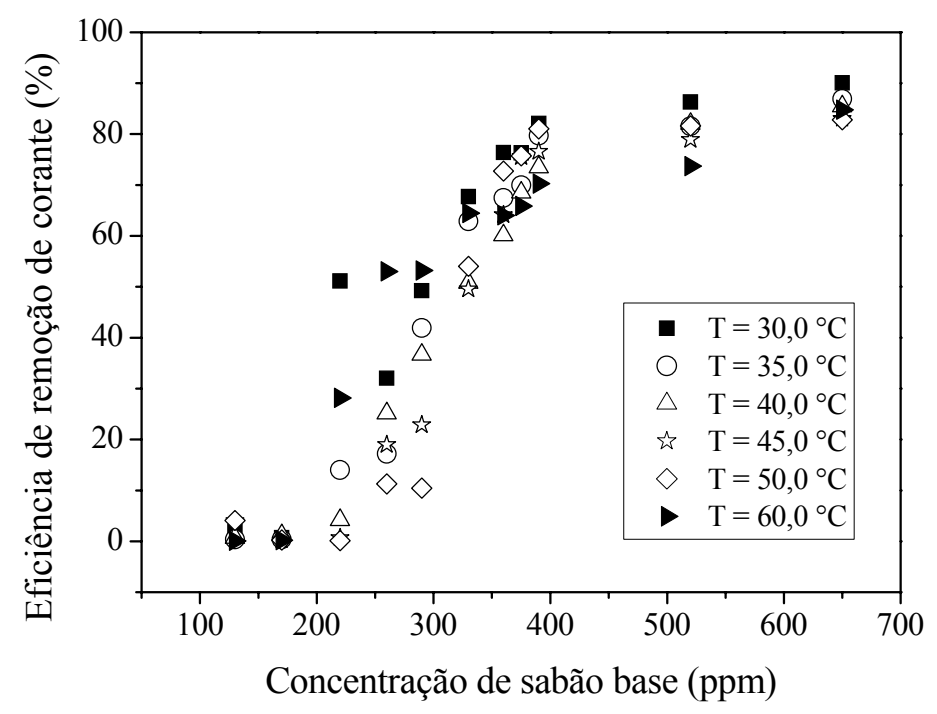

Figura 2 - Eficiência de remoção de corante para diferentes concentrações de tensoativo e temperatura.

A partir da Figura 2 observa-se que, para concentrações de tensoativo de 260 ppm abaixo, a 


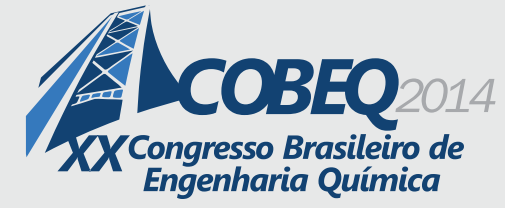

19 a 22 de outubro de 2014

Florianópolis/SC

eficiência de remoção de corante não foi tão elevada. Analisando as concentrações de 290 ppm ou mais nota-se que os resultados de remoção são mais significativos, atingindo no máximo $53,17 \%$ para temperatura de $60,0^{\circ} \mathrm{C}$. Os pontos nos quais a concentração de tensoativo foi igual ou superior a 390 ppm pode-se observar que a eficiência do processo tende a estabilizar em $90,0 \%$, demonstrando que a adição de tensoativo além de 650 ppm não resultará em ganho no processo.

Na região da Figura 2 que se encontra entre as concentrações de tensoativo de 220 e 390 ppm, é possível observar a elevação da eficiência de remoção para pequenas variações na concentração de tensoativo, sendo este comportamento o mesmo em todas as temperaturas. Além disso, no geral, a influência da temperatura existe, no entanto ela não é tão significativa ao ponto de poder ser comparada ao efeito da concentração de tensoativo, de forma que se pode afirmar uma independência do processo com relação a temperatura dentro da faixa estudada.

\subsection{Coeficiente de distribuição $(K)$}

A curva do coeficiente de distribuição em função da concentração de tensoativo mostrou praticamente o mesmo comportamento daquela para eficiência de remoção de corante, conforme observado na Figura 3. O coeficiente de distribuição permaneceu aproximadamente zero para concentrações de tensoativo baixas. O coeficiente de distribuição foi maior para concentrações de tensoativo de 520 e $650 \mathrm{ppm}$, alcançando um valor máximo de 9,68 para temperatura de $30,0{ }^{\circ} \mathrm{C}$ e concentração de tensoativo de 650 ppm.

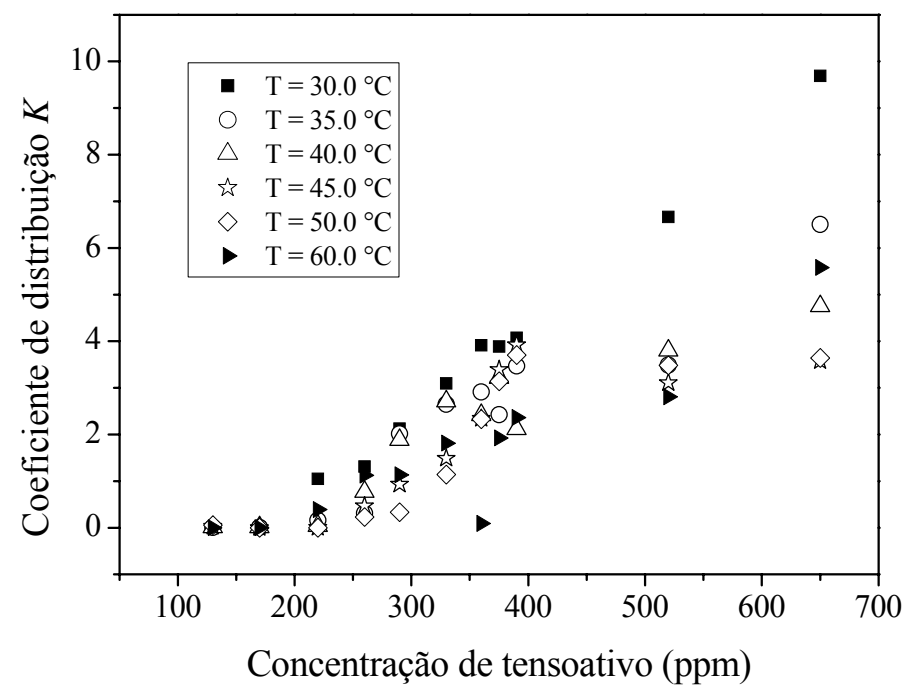

Figura 3 - Coeficiente de distribuição de corante para diferentes concentrações de tensoativo e temperatura.

Este valor de coeficiente de distribuição mostra a boa afinidade do corante pelo floco 
hidrofóbico. Diferente da remoção de corante o coeficiente de distribuição torna-se cada vez maior em concentrações elevadas, pois o denominador da Equação 2 diminui para próximo de zero nos casos de remoção de corante elevadas. Embora na Figura 3 a temperatura de $30,0^{\circ} \mathrm{C}$ apresente maior coeficiente de distribuição, estes foram bem próximos das demais temperaturas.

\subsection{Efeito do pH}

$\mathrm{O}$ pH foi avaliado, visto que a estabilidade do tensoativo está relacionada a este parâmetro. Medindo-se o pH das amostras, sem qualquer adição de uma solução básica ou ácida, este permaneceu em 9,3. Todas as amostras foram preparadas na temperatura de $30,0{ }^{\circ} \mathrm{C}$ em virtude do processo não ser tão dependente da temperatura e as concentrações de tensoativo utilizadas foram as que proporcionaram os melhores resultados de remoção na etapa anterior. A eficiência de remoção variou em função do $\mathrm{pH}$ conforme a Figura 4.

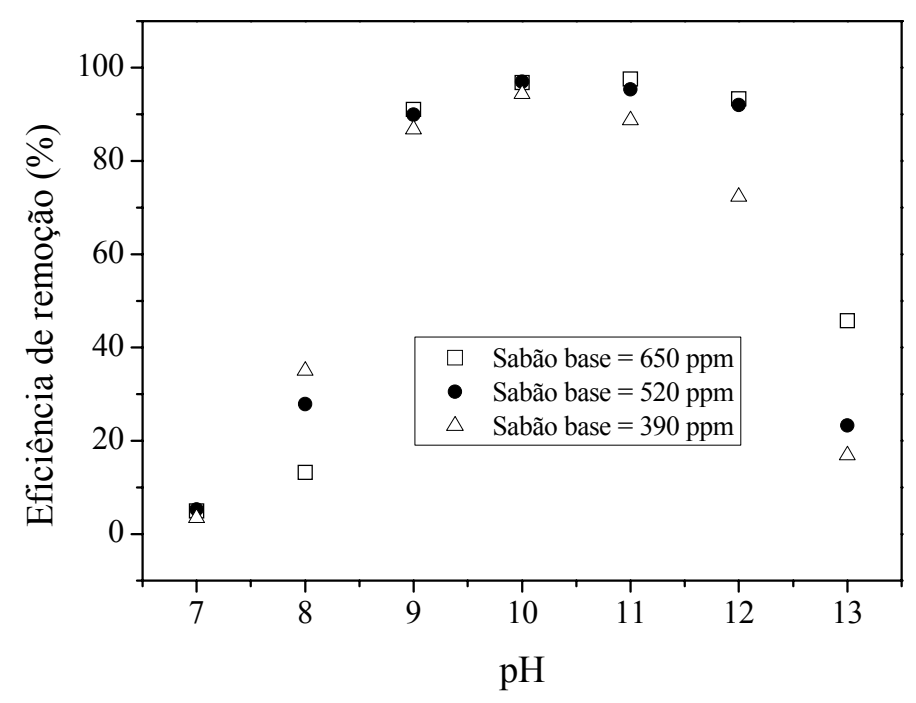

Figura 4 - Eficiência de remoção para diferentes pH.

Em pH 7 e 8 a remoção de corante do processo foi baixa devido a reação do sabão base com os prótons do ácido, diminuindo a reação com o cálcio e, consequentemente, reduzindo a formação dos flocos. Na faixa de pH de 9 a 12 a eficiência do processo é melhor em virtude da maior estabilidade do tensoativo, o que leva a formação de uma quantidade maior de flocos, que são responsáveis pela adsorção do corante. Além disso, a remoção de corante foi maior nos pontos em que a concentração de tensoativo foi maior, atingindo 97,58\% quando foi aplicado pH 11 e concentração de tensoativo de $650 \mathrm{ppm}$. 


\section{CONCLUSÃO}

A partir dos resultados obtidos pode-se concluir que a floculação micelar adsortiva é um processo eficiente para o tratamento de efluente contendo corante, alcançando 97,58\% de remoção. O efeito da concentração de tensoativo e $\mathrm{pH}$ mostraram-se significativos e, a temperatura afeta a remoção do corante de maneira menos considerável. O coeficiente de distribuição do processo alcançou 9,68 demonstrando a afinidade do corante pelo floco. A floculação micelar mostra-se como uma boa alternativa para o tratamento de efluentes contendo compostos orgânicos devido o caráter hidrofóbico do floco.

\section{NOMENCLATURA}

$C_{D Y 27}$ - Concentração de Direct Yellow 27

$K$ - coeficiente de distribuição

$m_{D Y 27}-$ massa de Direct Yellow 27

\section{REFERÊNCIAS BIBLIOGRÁFICAS}

ARSLAN-ALATON, I.; GURSOY, B. H.; SCHMIDT J. Advanced oxidation of acid and reactive dyes: Effect of Fenton treatment on aerobic, anoxic and anaerobic processes. Dyes and Pigments, v. 78, p. 117-130, 2008.

LAU, Y.; WONG, Y.; TENG, T.; MORAD, N.; RAFATULLAH, M.; ONG, S. Coagulationflocculation of azo dye Acid Orange 7 with green refined laterite soil. Chemical Engineering Journal, v. 246, p. 383-390, 2014.

MURUGANANDHAM, M.; SWAMINATHAN, M. Advanced oxidative decolourisation of Reactive Yellow 14 azo dye by $\mathrm{UV} / \mathrm{TiO}_{2}, \mathrm{UV} / \mathrm{H}_{2} \mathrm{O}_{2}, \mathrm{UV} / \mathrm{H}_{2} \mathrm{O}_{2} / \mathrm{Fe}^{2+}$ processes - a comparative study. Separation and Purification Technology, v. 48, p. 297-303, 2006.

PURKAIT, M. K.; DASGUPTA, S.; DE, S. Performance of TX-100 and TX-114 for the separation of chrysoidine dye using cloud point extraction. Journal of Hazardous Materials, v. B137, p. 827-835, 2006.

TEHRANI-BAGHA, A. R.; MAHMOODI, N. M.; MENGER, F. M. Degradation of a persistent organic dye from colored textile wastewater by ozonation. Desalination, v. 260, p. 34-38, 2010.

ZHANG, G.; YI, L.; DENG, H.; SUN, P. Dyes adsorption using a synthetic carboxymethyl celluloseacrylic acid adsorbent. Journal of Environmental Sciences, v. 26, p. 1203-1211, 2014. 